\section{MS37-04 Thermodynamics and kinetics of polymorphic transitions in amino acid crystals}

Joost A. van den Ende ${ }^{1}$, Mireille M.H. Smets ${ }^{1}$, Daniël T. de Jong ${ }^{1}$, Sander J.T. Brugman ${ }^{1}$, Bernd Ensing ${ }^{2}$, Paul T. Tinnemans ${ }^{1}$, Hugo Meekes ${ }^{1}$, Herma M. Cuppen

1. Institute for Molecules and Materials, Radboud University, Heyendaalseweg 135, 6525 AJ Nijmegen, The Netherlands

2. Van 't Hoff Institute for Molecular Sciences, University of Amsterdam, Science Park 904, 1098 XH Amsterdam, The Netherlands

email: j.vandenende@science.ru.nl

Polymorphism in molecular crystals can effect bioavailability of pharmaceuticals through a difference in solubility or dissolution rate between the polymorphic forms. To achieve optimal control of polymorphism it is essential to fully understand both the thermodynamics and kinetics of transitions between the different polymorphic forms. We will present a combined experimental and computational study of these relations for DL-norleucine and some preliminary computational results for L-phenylalanine. Both compounds crystallize in forms that exhibit hydrogen-bonded bilayers. The mechanism of solid-to-solid polymorphic transitions in these crystals is likely to be governed by cooperative movement of full bilayers.

For the $\alpha$ and $\beta$ polymorphic forms of DL-norleucine the temperature dependence of the lattice parameters and the energetic properties in Molecular Dynamics (MD) simulations is remarkably similar.[1,2] These results are confirmed by single-crystal X-ray diffraction and Differential Scanning Calorimetry (DSC) measurements.[2] The absence of any discontinuities in the properties opens the possibility of a second-order solid-to-solid polymorphic transition between these two enantiotropically related polymorphs. Nudged Elastic Band (NEB) calculations[2], however, show the existence of relatively large energy barriers upon transforming the $\beta$ polymorphic form towards the $\alpha$ form, which indicates a first-order transition.

References:

[1] J. A. van den Ende and H. M. Cuppen, Cryst. Growth Des., 2014, 14, 3343-3351, dx.doi.org/10.1021/cg5002804

[2] J. A. van den Ende, M. M. H. Smets, D. T. de Jong, S. J. T. Brugman, B. Ensing, P. T. Tinnemans, H. Meekes, and H. M. Cuppen, Faraday. Discuss., accepted for publication, DOI: 10.1039/c4fd00214h
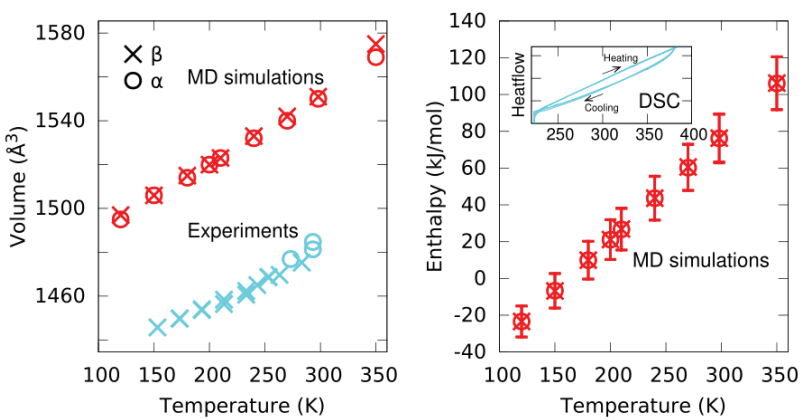

Figure 1. The temperature dependence of the volume and energetic properties of the $\alpha$ and $\beta$ polymorphic forms of DL-norleucine is remarkably similar, both in MD-simulations and experiments.

Keywords: polymorphism, solid-to-solid phase transitions, Molecular Dynamics simulations, amino acids 